CUBO A Mathematical Journal Vol.12, $N^{\underline{O}}$ 02, (261-274). June 2010

\title{
Real and stable ranks for certain crossed products of Toeplitz algebras
}

\author{
TAKAHIRO SUdO \\ Department of Mathematical Sciences, \\ Faculty of Science, University of the Ryukyus, \\ Nishihara, Okinawa 903-0213, Japan \\ email: sudo@math.u-ryukyu.ac.jp
}

\begin{abstract}
We consider the algebraic structure of certain crossed products of the Toeplitz algebra and its tensor products. Using the structure, we estimate the stable rank and real rank of those crossed products. In particular, we obtain a real rank estimate for extensions of $C^{*}$-algebras.
\end{abstract}

\section{RESUMEN}

Consideramos la estructura algebraica de ciertos productos cruzados de algebra de Toeplitz y sus productos tensoriales. Usando la estructura estimamos el rango estable y el rango real de estos productos cruzados. En particular, obtenemos una estimativa del rango real para extensiones de $C^{*}$-algebras.

Key words and phrases: $C^{*}$-algebra, Crossed products, Stable rank, Real rank, Toeplitz algebra.

2000 Math. Subj. Class.: Primary 46L05, $46 L 80$

\section{Introduction}

Crossed products of $C^{*}$-algebras (by automorphisms) have been very interesting research objects in the $C^{*}$-algebra theory. See [9] as a reference. As well, crossed products of $C^{*}$-algebras by endomorphisms 
have been studied (rather recently). A typical and important example is given by the rotation $C^{*}$ algebra, that is defined as the crossed product of $C(\mathbb{T})$ by the rotation action of the group $\mathbb{Z}$ of integers, where $C(\mathbb{T})$ is the $C^{*}$-algebra of all continuous functions on the 1 -torus $\mathbb{T}$, and is also the universal $C^{*}$-algebra generated by a unitary. More generally, noncommutative tori are defined as successive crossed products by $\mathbb{Z}$. On the other hand, another example is given by the group $C^{*}$-algebra of the semi-direct product $\mathbb{Z}^{n} \rtimes \mathbb{Z}$, that is viewed as the crossed product of $C\left(\mathbb{T}^{n}\right)$ by the adjoint action of $\mathbb{Z}$, where $\mathbb{T}^{n}$ is the $n$-torus. More generally, the group $C^{*}$-algebras of successive semi-direct products by $\mathbb{Z}$ are viewed as successive crossed products by $\mathbb{Z}$.

Our first motivation is to replace $C(\mathbb{T})$ with the Toeplitz algebra $\mathfrak{F}$, that is the universal $C^{*}$ algebra generated by an isometry, and replace $\mathbb{Z}$ with the semigroup $\mathbb{N}$ of natural numbers (with zero), and consider the crossed product of $\mathfrak{F}$ by $\mathbb{N}$. Furthermore, we replace $C\left(\mathbb{T}^{n}\right)$ with $\otimes^{n} \mathfrak{F}$ the $n$ fold tensor product of $\mathfrak{F}$ and consider the crossed product of $\otimes^{n} \mathfrak{F}$ by $\mathbb{N}$. While the crossed products of $C\left(\mathbb{T}^{k}\right)$ by $\mathbb{Z}$, that are viewed as noncommutative manifolds, have been studied well, the replacements by isometries: the crossed products of $\otimes^{n} \mathfrak{F}$ by $\mathbb{N}$, have not been studied explicitly yet.

Under those circumstances, in this paper we study certain crossed products of the Toeplitz algebra and its tensor products. The algebraic structure of those crossed products is given explicitly (and inductively) in Section 1. It is found that the crossed products have quotients that are isomorphic to the group $C^{*}$-algebras of generalized discrete $a x+b$ groups that are defined and studied in [14], so that they may be viewed as the $C^{*}$-algebras of generalized discrete $a x+b$ semigroups in a sense. (Our first effort was to find such an analogue to the group $C^{*}$-algebras of the Heisenberg discrete group, but this has not been successful yet.) The K-theory groups of the crossed products are computed by using the Pimsner-Voiculescu exact sequence. Using the structure (and in part the K-theory results) obtained, we estimate the stable rank and connected stable rank of the crossed products in Section 1 , and estimate their real rank as well as the real rank of the group $C^{*}$-algebras of the generalized discrete $a x+b$ groups in Section 2. Note that the stable and real ranks are viewed as noncommutative complex and real dimensions respectively. For estimating the real rank, we obtain a new real rank estimate for extensions of $C^{*}$-algebras. It turns out that this estimate is quite useful for estimating and determining the real rank of extensions of $C^{*}$-algebras. The stable rank, connected stable rank, and real rank formulae obtained for those crossed products and the real rank formulae for those group $C^{*}$-algebras are new, and the ranks are estimated with the dimension of the spaces of 2-dimensional irreducible representations that correspond to certain subquotients of the group $C^{*}$-algebras. In addition, a partial duality result on crossed products of $C^{*}$-algebras by $\mathbb{N}$ is obtained, which may be of some independent interest and would be useful for further research in a direction.

Notation. We denote by $\operatorname{sr}(\mathfrak{A})$ the stable rank of a (unital) $C^{*}$-algebra $\mathfrak{A}$, and by $\operatorname{csr}(\mathfrak{A})$ its connected stable rank. By definition, $\operatorname{sr}(\mathfrak{A}) \leq n$ if and only if $L_{n}(\mathfrak{A})$ is dense in $\mathfrak{A}^{n}$, where $\left(a_{j}\right) \in L_{n}(\mathfrak{A})$ if there exists $\left(b_{j}\right) \in \mathfrak{A}^{n}$ such that $\sum_{j=1}^{n} b_{j} a_{j}=1 \in \mathfrak{A}$. Also, $\operatorname{csr}(\mathfrak{A}) \leq n$ if and only if $L_{m}(\mathfrak{A})$ is connected for any $m \geq n$. Refer to [10]. We denote by $\operatorname{RR}(\mathfrak{A})$ the real rank of $\mathfrak{A}$. By definition, $\operatorname{RR}(\mathfrak{A}) \leq n-1$ if and only if $L_{n}(\mathfrak{A})_{s a}$ is dense in $\left(\mathfrak{A}_{s a}\right)^{n}$, where $L_{n}(\mathfrak{A})_{s a}$ and $\mathfrak{A}_{s a}$ are the sets of all self-adjoint elements of $L_{n}(\mathfrak{A})$ and $\mathfrak{A}$ respectively. Refer to [3].

Recall from [14] that the generalized discrete $a x+b$ group that is a semi-direct product $\mathbb{Z}^{n} \rtimes \mathbb{Z}$ 
is defined by the following $(n+1) \times(n+1)$ matrices:

$$
\left(\begin{array}{cc}
\oplus^{n} e^{\pi i t} & s \\
0_{n} & 1
\end{array}\right) \in G L_{n+1}(\mathbb{Z}),
$$

where $\oplus^{n} e^{\pi i t}$ means the $n \times n$ diagonal matrix with diagonal entries $e^{\pi i t}$ for $t \in \mathbb{Z}$, and $s \in \mathbb{Z}^{n}$ (a column vector), and $0_{n}=(0, \cdots, 0) \in \mathbb{Z}^{n}$ (a row vector).

\section{Structure and Stable rank}

Let $\mathfrak{F}$ be the Toeplitz algebra, that is defined to be the universal $C^{*}$-algebra generated by a (proper) isometry $s$. Write $\mathfrak{F}=C^{*}(s)$.

Definition 2.1. We define the crossed product of $\mathfrak{F}$ by an action of $\mathbb{N}$ to be the universal $C^{*}$-algebra generated by $\mathfrak{F}$ and an isometry $t$ such that the action $\alpha$ of $\mathbb{N}$ on $\mathfrak{F}$ is given by $\alpha_{1}(x)=t x t^{*}$ for $x \in \mathfrak{F}$. Denote it by $C^{*}\left(H_{1,1}\right)=\mathfrak{F} \rtimes_{\alpha} \mathbb{N}$ and call it the $C^{*}$-algebra of the discrete $a x+b$ semigroup $H_{1,1}$ since $\mathfrak{F} \cong C^{*}(\mathbb{N})$ the $C^{*}$-algebra of $\mathbb{N}$, so that we may write $H_{1,1}=\mathbb{N} \rtimes \mathbb{N}$ just as a symbol like a semi-direct product.

It is well known that $\mathfrak{F}$ has the decomposition into the exact sequence:

$$
0 \rightarrow \mathbb{K} \rightarrow \mathfrak{F} \rightarrow C(\mathbb{T}) \rightarrow 0
$$

where $\mathbb{K}$ is the $C^{*}$-algebra of compact operators on a separable Hilbert space. Furthermore, this $\mathbb{K}$ is isomorphic to the commutator ideal of $\mathfrak{F}$. Refer to [6].

Theorem 2.2. The $C^{*}$-algebra $C^{*}\left(H_{1,1}\right)=\mathfrak{F} \rtimes_{\alpha} \mathbb{N}$ has the decomposition into the exact sequence:

$$
0 \rightarrow \mathbb{K} \rtimes_{\alpha} \mathbb{N} \rightarrow \mathfrak{F} \rtimes_{\alpha} \mathbb{N} \rightarrow C(\mathbb{T}) \rtimes_{\alpha} \mathbb{N} \rightarrow 0,
$$

Moreover, $\mathbb{K} \rtimes_{\alpha} \mathbb{N} \cong \mathbb{K} \otimes C(\mathbb{T})$ and $C(\mathbb{T}) \rtimes_{\alpha} \mathbb{N} \cong C(\mathbb{T}) \rtimes_{\alpha} \mathbb{Z}$ a crossed product of $C(\mathbb{T})$ by a unitary action of $\mathbb{Z}$, which is isomorphic to the group $C^{*}$-algebra of the discrete ax $+b$ group $\mathbb{Z} \rtimes \mathbb{Z}$.

Proof. Let $x \in \mathfrak{F}=C^{*}(s)$. Since $x x^{*}-x^{*} x$ is a compact operator, $t\left(x x^{*}-x^{*} x\right) t^{*}=\left(t x t^{*}\right)\left(t x^{*} t^{*}\right)-$ $\left(t x^{*} t^{*}\right)\left(t x t^{*}\right)$ is also compact. Therefore, $\mathbb{K}$ is invariant under the action $\alpha$ of $\mathbb{N}$. Hence we obtain the exact sequence.

Furthermore, we have

$$
\mathbb{K} \rtimes_{\alpha} \mathbb{N} \cong \mathbb{K} \rtimes_{\alpha} \mathbb{Z} \cong \mathbb{K} \otimes C(\mathbb{T}),
$$

where the first isomorphism follows from that the action $\alpha$ on $\mathbb{K}$ is an automorphism as discussed above, and the second isomorphism follows from that any automorphism on $\mathbb{K}$ is implemented by a unitary, i.e. an adjoint action by a unitary, so that $\mathbb{K} \rtimes_{\alpha} \mathbb{Z} \cong \mathbb{K} \otimes C^{*}(\mathbb{Z})$, where $C^{*}(\mathbb{Z})$ is the group $C^{*}$-algebra of $\mathbb{Z}$, that is isomorphic to $C(\mathbb{T})$ by the Fourier transform.

Also, we have $C(\mathbb{T}) \rtimes_{\alpha} \mathbb{N} \cong C(\mathbb{T}) \rtimes_{\alpha} \mathbb{Z}$ since the action $\alpha$ on $C(\mathbb{T})$ by $\mathbb{N}$ must be an automorphism implemented by a unitary, which is isomorphic to the group $C^{*}$-algebra of the discrete $a x+b$ group $\mathbb{Z} \rtimes \mathbb{Z}$. 
Remark. We may view this extension property as the definition for $C^{*}\left(H_{1,1}\right)=\mathfrak{F} \rtimes_{\alpha} \mathbb{N}$. By universality, there is a quotient map from $\mathfrak{F} \rtimes_{\alpha} \mathbb{N}$ to $C(\mathbb{T}) \rtimes_{\alpha} \mathbb{Z}$. The similar remark as this can be made for the structure results given below.

Proposition 2.3. The K-theory groups of $C^{*}\left(H_{1,1}\right)$ are obtained as:

$$
K_{j}\left(C^{*}\left(H_{1,1}\right)\right) \cong \mathbb{Z} \quad(j=0,1) .
$$

Proof. Since $C^{*}\left(H_{1,1}\right)=\mathfrak{F} \rtimes_{\alpha} \mathbb{N}$, we have the Pimsner-Voiculescu exact sequence of K-groups for crossed products of $C^{*}$-algebras by $\mathbb{N}$ (as well as $\left.\mathbb{Z}\right)$ :

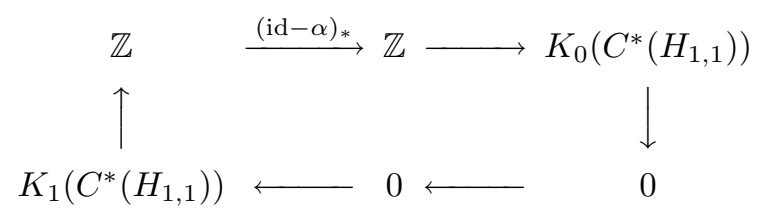

(see [12] and [2]), where $K_{0}(\mathfrak{F}) \cong \mathbb{Z}$ and $K_{1}(\mathfrak{F}) \cong 0$. Since the map (id $\left.-\alpha\right)_{*}$ is trivial, where id is the identity map on $\mathfrak{F}$, we obtain $K_{j}\left(C^{*}\left(H_{1,1}\right)\right) \cong \mathbb{Z}$ for $j=0,1$.

Remark. The six-term exact sequence for the exact sequence obtained above is

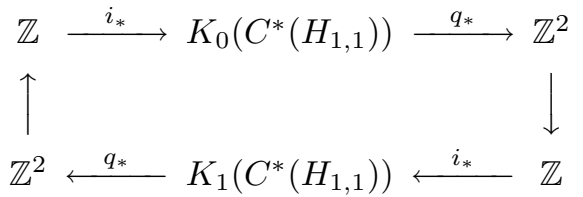

where $K_{j}(\mathbb{K} \otimes C(\mathbb{T})) \cong K_{j}(C(\mathbb{T})) \cong \mathbb{Z}$ for $j=0,1$, and $K_{j}\left(C(\mathbb{T}) \rtimes_{\alpha} \mathbb{Z}\right) \cong \mathbb{Z}^{2}(j=0,1)$ by the (usual) Pimsner-Voiculescu exact sequence. Consequently, the maps $i_{*}$ induced by the inclusion $i: \mathbb{K} \otimes C(\mathbb{T}) \rightarrow$ $C^{*}\left(H_{1,1}\right)$ are zero, so that the maps $q_{*}$ induced by the quotient map $q: C^{*}\left(H_{1,1}\right) \rightarrow C(\mathbb{T}) \rtimes_{\alpha} \mathbb{Z}$ are injective.

Theorem 2.4. The stable rank of $C^{*}\left(H_{1,1}\right)$ is 2 . The connected stable rank of $C^{*}\left(H_{1,1}\right)$ is 2 .

Proof. By [10, Theorems 4.3, 4.4, and 4.11], we have the following estimates:

$$
\begin{aligned}
& \operatorname{sr}\left(C^{*}\left(H_{1,1}\right)\right) \leq \max \{\operatorname{sr}(\mathbb{K} \otimes C(\mathbb{T})), \operatorname{sr}(C(\mathbb{T}) \rtimes \mathbb{Z}), \operatorname{csr}(C(\mathbb{T}) \rtimes \mathbb{Z})\}, \\
& \text { and } \max \{\operatorname{sr}(\mathbb{K} \otimes C(\mathbb{T})), \operatorname{sr}(C(\mathbb{T}) \rtimes \mathbb{Z})\} \leq \operatorname{sr}\left(C^{*}\left(H_{1,1}\right)\right) .
\end{aligned}
$$

Furthermore, by [10, Theorems 3.6 and 6.4 and Proposition 1.7] $\operatorname{sr}(\mathbb{K} \otimes C(\mathbb{T}))=\operatorname{sr}(C(\mathbb{T}))=1$. Note that $C(\mathbb{T}) \rtimes \mathbb{Z} \cong C^{*}(\mathbb{Z} \rtimes \mathbb{Z})$. By the stable rank and connected stable rank formulae in [14, Remark 3.4 with a correction (see the remark below) we have

$$
\operatorname{sr}\left(C^{*}(\mathbb{Z} \rtimes \mathbb{Z})\right)=2, \quad \text { and } \quad \operatorname{csr}\left(C^{*}(\mathbb{Z} \rtimes \mathbb{Z})\right) \leq 2 .
$$

The same estimates (from above, $\leq 2$ ) for $C(\mathbb{T}) \rtimes \mathbb{Z}$ are also obtained by using $[10$, Theorem 7.1 and Corollary 8.6].

On the other hand, by [13, Theorem 3.9] we have

$$
\operatorname{csr}\left(C^{*}\left(H_{1,1}\right)\right) \leq \max \{\operatorname{csr}(\mathbb{K} \otimes C(\mathbb{T})), \operatorname{csr}(C(\mathbb{T}) \rtimes \mathbb{Z})\} .
$$


By [13, Theorem 3.10], $\operatorname{csr}(\mathbb{K} \otimes C(\mathbb{T})) \leq 2$. Since $K_{1}$-group of $C^{*}\left(H_{1,1}\right)$ is not trivial as shown above, we have $\operatorname{csr}\left(C^{*}\left(H_{1,1}\right)\right) \geq 2$ (cf. [4, Corollary 1.6]).

Remark. The stable rank estimate in [14, Remark 3.4] after a correction is

$$
\begin{aligned}
& \operatorname{sr}\left(C_{0}\left(\mathbb{R}^{n+1}\right) \otimes M_{2}(\mathbb{C})\right)=\lceil\lfloor(n+1) / 2\rfloor / 2\rceil+1 \leq \\
& \operatorname{sr}\left(C^{*}\left(\mathbb{Z}^{n} \rtimes \mathbb{Z}\right)\right) \leq \operatorname{csr}\left(C_{0}\left(\mathbb{R}^{n+1}\right) \otimes M_{2}(\mathbb{C})\right) \leq\lceil\lfloor(n+2) / 2\rfloor / 2\rceil+1,
\end{aligned}
$$

and the connected stable rank estimate in it after that is

$$
\operatorname{csr}\left(C^{*}\left(\mathbb{Z}^{n} \rtimes \mathbb{Z}\right)\right) \leq \operatorname{csr}\left(C_{0}\left(\mathbb{R}^{n+1}\right) \otimes M_{2}(\mathbb{C})\right) \leq\lceil\lfloor(n+2) / 2\rfloor / 2\rceil+1,
$$

where $C^{*}\left(\mathbb{Z}^{n} \rtimes \mathbb{Z}\right)$ is the group $C^{*}$-algebra of the generalized $a x+b$ group defined in [14], and $\lfloor x\rfloor$ means the maximum integer $\leq x$, and $\lceil y\rceil$ is the least integer $\geq y$. In particular, if $n$ is odd, then $\operatorname{sr}\left(C^{*}\left(\mathbb{Z}^{n} \rtimes \mathbb{Z}\right)\right)=\lceil\lfloor(n+1) / 2\rfloor / 2\rceil+1$. Furthermore, if $n=4 m$, then the inequality does not become equality, but if $n=4 m+2$, then the inequality becomes equality. As for the correction, in fact, $C_{0}\left(\mathbb{R}^{n-j+1}\right)$ in [14, Theorem 3.3] should have been replaced with $C_{0}\left(\mathbb{R}^{n-j+2}\right)(1 \leq j \leq n)$.

As a note, the Toeplitz algebra $\mathfrak{F}$ has stable rank 2 and connected stable rank $\leq 2$. This follows from using [10], [8], and [13] as above, where the result of [8] says that if the index map in the six-term exact sequence of $\mathrm{K}$-groups for a $C^{*}$-algebra extension $E$ is nonzero, then $E$ can not have stable rank 1.

Let $\mathfrak{F} \otimes \mathfrak{F}$ be the $C^{*}$-tensor product of $\mathfrak{F}$, which is also defined to be the universal $C^{*}$-algebra generated by $*$-commuting isometries $s_{1}, s_{2}$, which means that each $s_{j}$ commutes with both $s_{i}$ and $s_{i}^{*}(i \neq j)$.

Definition 2.5. We define the $C^{*}$-algebra of the (generalized) $a x+b$ semigroup $H_{2,1}=\mathbb{N}^{2} \rtimes \mathbb{N}$ (just as a symbol like a semi-direct product) to be the universal $C^{*}$-algebra generated by $\mathfrak{F} \otimes \mathfrak{F}$ and an isometry $t \otimes t$ such that the (product) action $\alpha \otimes \alpha$ of $\mathbb{N}$ on $\mathfrak{F} \otimes \mathfrak{F}$ is given by $(\alpha \otimes \alpha)_{1}(x \otimes y)=$ $(t \otimes t)(x \otimes y)(t \otimes t)^{*}=t x t^{*} \otimes t y t^{*}$ for $x \otimes y \in \mathfrak{F} \otimes \mathfrak{F}$. Denote it by $C^{*}\left(H_{2,1}\right)=(\mathfrak{F} \otimes \mathfrak{F}) \rtimes_{\alpha \otimes \alpha} \mathbb{N}$ the crossed product of $\mathfrak{F} \otimes \mathfrak{F}$ by $\alpha \otimes \alpha$ of $\mathbb{N}$.

In what follows, we often omit the symbol for actions in crossed products.

Proposition 2.6. The $C^{*}$-algebra $C^{*}\left(H_{2,1}\right)=(\mathfrak{F} \otimes \mathfrak{F}) \rtimes_{\alpha \otimes \alpha} \mathbb{N}$ has the structure as follows:

$$
0 \rightarrow(\mathfrak{F} \otimes \mathbb{K}) \rtimes \mathbb{N} \rightarrow C^{*}\left(H_{2,1}\right) \rightarrow(\mathfrak{F} \otimes C(\mathbb{T})) \rtimes \mathbb{N} \rightarrow 0
$$

and the quotient and closed ideal have the decompositions as follows:

$$
\begin{aligned}
0 & \rightarrow(\mathbb{K} \otimes C(\mathbb{T})) \rtimes \mathbb{N} \rightarrow(\mathfrak{F} \otimes C(\mathbb{T})) \rtimes \mathbb{N} \rightarrow C\left(\mathbb{T}^{2}\right) \rtimes \mathbb{N} \rightarrow 0, \\
\text { and } \quad & 0 \rightarrow(\mathbb{K} \otimes \mathbb{K}) \rtimes \mathbb{N} \rightarrow(\mathfrak{F} \otimes \mathbb{K}) \rtimes \mathbb{N} \rightarrow(C(\mathbb{T}) \otimes \mathbb{K}) \rtimes \mathbb{N} \rightarrow 0 .
\end{aligned}
$$

Furthermore, $(\mathbb{K} \otimes C(\mathbb{T})) \rtimes \mathbb{N} \cong \mathbb{K} \otimes(C(\mathbb{T}) \rtimes \mathbb{Z}),(C(\mathbb{T}) \otimes \mathbb{K}) \rtimes \mathbb{N} \cong(C(\mathbb{T}) \rtimes \mathbb{Z}) \otimes \mathbb{K}$, and $(\mathbb{K} \otimes \mathbb{K}) \rtimes \mathbb{N} \cong$ $\mathbb{K} \otimes C(\mathbb{T})$, and $C\left(\mathbb{T}^{2}\right) \rtimes \mathbb{N} \cong C\left(\mathbb{T}^{2}\right) \rtimes \mathbb{Z}$, which is isomorphic to the group $C^{*}$-algebra of the (generalized) discrete $a x+b$ group $\mathbb{Z}^{2} \rtimes \mathbb{Z}$. 
Proof. The quotient and closed ideal, and their decompositions are deduced from the invariance of the action $\alpha \otimes \alpha$. Note that

$$
\begin{aligned}
& (\mathbb{K} \otimes C(\mathbb{T})) \rtimes \mathbb{N} \cong(\mathbb{K} \otimes C(\mathbb{T})) \rtimes \mathbb{Z}, \quad \text { and } \\
& (C(\mathbb{T}) \otimes \mathbb{K}) \rtimes \mathbb{N} \cong(C(\mathbb{T}) \otimes \mathbb{K}) \rtimes \mathbb{Z} .
\end{aligned}
$$

Furthermore, $(\mathbb{K} \otimes \mathbb{K}) \rtimes \mathbb{N}$ is isomorphic to the following:

$$
(\mathbb{K} \otimes \mathbb{K}) \rtimes \mathbb{Z} \cong(\mathbb{K} \otimes \mathbb{K}) \otimes C(\mathbb{T}) \cong \mathbb{K} \otimes C(\mathbb{T})
$$

Proposition 2.7. The K-theory groups of $C^{*}\left(H_{2,1}\right)$ are obtained as:

$$
K_{j}\left(C^{*}\left(H_{2,1}\right)\right) \cong \mathbb{Z} \quad(j=0,1) .
$$

Proof. Since $C^{*}\left(H_{2,1}\right)=(\mathfrak{F} \otimes \mathfrak{F}) \rtimes_{\alpha} \mathbb{N}$, we have the Pimsner-Voiculescu sequence:

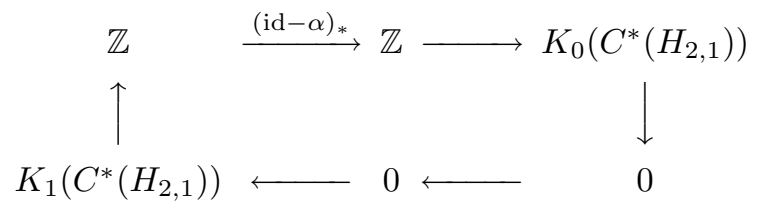

where $K_{0}(\mathfrak{F} \otimes \mathfrak{F}) \cong \mathbb{Z}$ and $K_{1}(\mathfrak{F} \otimes \mathfrak{F}) \cong 0$ by the Künneth formula (see [2]). Since the map (id $\left.-\alpha\right)_{*}$ is trivial, where id is the identity map on $\otimes^{2} \mathfrak{F}$, we obtain $K_{j}\left(C^{*}\left(H_{2,1}\right)\right) \cong \mathbb{Z}$ for $j=0,1$.

Theorem 2.8. The stable rank of $C^{*}\left(H_{2,1}\right)$ is 2 . The connected stable rank of $C^{*}\left(H_{2,1}\right)$ is 2 .

Proof. By [10, Theorems 4.3, 4.4, and 4.11], we have the following estimates:

$$
\begin{aligned}
& \operatorname{sr}\left(C^{*}\left(H_{2,1}\right)\right) \leq \max \{\operatorname{sr}((\mathfrak{F} \otimes \mathbb{K}) \rtimes \mathbb{N}), \operatorname{sr}((\mathfrak{F} \otimes C(\mathbb{T})) \rtimes \mathbb{N}), \operatorname{csr}((\mathfrak{F} \otimes C(\mathbb{T})) \rtimes \mathbb{N})\}, \\
& \text { and } \quad \max \{\operatorname{sr}((\mathfrak{F} \otimes \mathbb{K}) \rtimes \mathbb{N}), \operatorname{sr}((\mathfrak{F} \otimes C(\mathbb{T})) \rtimes \mathbb{N})\} \leq \operatorname{sr}\left(C^{*}\left(H_{2,1}\right)\right),
\end{aligned}
$$

and moreover,

$$
\begin{array}{ll} 
& \operatorname{sr}((\mathfrak{F} \otimes C(\mathbb{T})) \rtimes \mathbb{N}) \leq \\
& \max \left\{\operatorname{sr}(\mathbb{K} \otimes(C(\mathbb{T}) \rtimes \mathbb{Z})), \operatorname{sr}\left(C\left(\mathbb{T}^{2}\right) \rtimes \mathbb{Z}\right), \operatorname{csr}\left(C\left(\mathbb{T}^{2}\right) \rtimes \mathbb{Z}\right)\right\}, \\
\text { and } & \max \left\{\operatorname{sr}(\mathbb{K} \otimes(C(\mathbb{T}) \rtimes \mathbb{Z})), \operatorname{sr}\left(C\left(\mathbb{T}^{2}\right) \rtimes \mathbb{Z}\right)\right\} \leq \operatorname{sr}((\mathfrak{F} \otimes C(\mathbb{T})) \rtimes \mathbb{N}), \\
\text { and } & \operatorname{sr}((\mathfrak{F} \otimes \mathbb{K}) \rtimes \mathbb{N}) \leq \\
& \max \{\operatorname{sr}(\mathbb{K} \otimes C(\mathbb{T})), \operatorname{sr}((C(\mathbb{T}) \rtimes \mathbb{Z}) \otimes \mathbb{K}), \operatorname{csr}((C(\mathbb{T}) \rtimes \mathbb{Z}) \otimes \mathbb{K})\}, \\
\text { and } & \max \{\operatorname{sr}(\mathbb{K} \otimes C(\mathbb{T})), \operatorname{sr}((C(\mathbb{T}) \rtimes \mathbb{Z}) \otimes \mathbb{K})\} \leq \operatorname{sr}((\mathfrak{F} \otimes \mathbb{K}) \rtimes \mathbb{N}) .
\end{array}
$$

Furthermore, by [10, Theorems 3.6 and 6.4] $\operatorname{sr}(\mathbb{K} \otimes(C(\mathbb{T}) \rtimes \mathbb{Z}))=\operatorname{sr}(C(\mathbb{T}) \rtimes \mathbb{Z})=2$. Note that $C\left(\mathbb{T}^{2}\right) \rtimes \mathbb{Z} \cong C^{*}\left(\mathbb{Z}^{2} \rtimes \mathbb{Z}\right)$. By the stable rank and connected stable rank formulae in [14, Remark 3.4] with a correction (see the remark above) we have

$$
\operatorname{sr}\left(C^{*}\left(\mathbb{Z}^{2} \rtimes \mathbb{Z}\right)\right)=2, \quad \text { and } \quad \operatorname{csr}\left(C^{*}\left(\mathbb{Z}^{2} \rtimes \mathbb{Z}\right)\right) \leq 2 .
$$


On the other hand, by [13, Theorem 3.9] we have

$$
\operatorname{csr}\left(C^{*}\left(H_{2,1}\right)\right) \leq \max \{\operatorname{csr}((\mathfrak{F} \otimes \mathbb{K}) \rtimes \mathbb{N}), \operatorname{csr}((\mathfrak{F} \otimes C(\mathbb{T})) \rtimes \mathbb{N})\},
$$

and moreover,

$$
\begin{array}{ll} 
& \operatorname{csr}((\mathfrak{F} \otimes \mathbb{K}) \rtimes \mathbb{N}) \leq \\
& \max \left\{\operatorname{csr}(\mathbb{K} \otimes(C(\mathbb{T}) \rtimes \mathbb{Z})), \operatorname{csr}\left(C\left(\mathbb{T}^{2}\right) \rtimes \mathbb{Z}\right)\right\} \leq 2, \\
\text { and } \quad & \operatorname{csr}((\mathfrak{F} \otimes C(\mathbb{T})) \rtimes \mathbb{N}) \leq \\
& \max \{\operatorname{csr}(\mathbb{K} \otimes C(\mathbb{T})), \operatorname{csr}((C(\mathbb{T}) \rtimes \mathbb{Z}) \otimes \mathbb{K})\} \leq 2 .
\end{array}
$$

Hence, it follows that $\operatorname{csr}\left(C^{*}\left(H_{2,1}\right)\right) \leq 2$. Therefore, $\operatorname{sr}\left(C^{*}\left(H_{2,1}\right)\right)=2$ is obtained from the first part of this proof. Since $K_{1}$-group of $C^{*}\left(H_{2,1}\right)$ is not trivial as shown above, we have $\operatorname{csr}\left(C^{*}\left(H_{2,1}\right)\right) \geq 2$ (cf. [4, Corollary 1.6]).

Let $\otimes^{n} \mathfrak{F}$ be the $n$-fold $C^{*}$-tensor product of $\mathfrak{F}$, which is also defined to be the universal $C^{*}$-algebra generated by $n *$-commuting isometries $s_{j}(1 \leq j \leq n)$, which means that each $s_{j}$ commutes with both $s_{i}$ and $s_{i}^{*}$ for any $i \neq j$.

Definition 2.9. We define the $C^{*}$-algebra of the (generalized) $a x+b$ semigroup $H_{n, 1}=\mathbb{N}^{n} \rtimes \mathbb{N}$ (just as a symbol like a semi-direct product) to be the universal $C^{*}$-algebra generated by $\otimes^{n} \mathfrak{F}$ and an isometry $\otimes^{n} t$ such that the (product) action $\otimes^{n} \alpha$ of $\mathbb{N}$ on $\otimes^{n} \mathfrak{F}$ is given by $\left(\otimes^{n} \alpha\right)_{1}\left(\otimes_{j=1}^{n} x_{j}\right)=$ $\left(\otimes^{n} t\right)\left(\otimes_{j=1}^{n} x_{j}\right)\left(\otimes^{n} t\right)^{*}=\otimes_{j=1}^{n} t x_{j} t^{*}$ for $\otimes_{j=1}^{n} x_{j} \in \otimes^{n} \mathfrak{F}$. Denote it by $C^{*}\left(H_{n, 1}\right)=\left(\otimes^{n} \mathfrak{F}\right) \rtimes_{\otimes^{n} \alpha} \mathbb{N}$ the crossed product of $\otimes^{n} \mathfrak{F}$ by $\otimes^{n} \alpha$ of $\mathbb{N}$.

Proposition 2.10. The $C^{*}$-algebra $C^{*}\left(H_{n, 1}\right)=\left(\otimes^{n} \mathfrak{F}\right) \rtimes_{\otimes^{n} \alpha} \mathbb{N}$ has the structure:

$$
0 \rightarrow\left(\left(\otimes^{n-1} \mathfrak{F}\right) \otimes \mathbb{K}\right) \rtimes \mathbb{N} \rightarrow C^{*}\left(H_{n, 1}\right) \rightarrow\left(\left(\otimes^{n-1} \mathfrak{F}\right) \otimes C(\mathbb{T})\right) \rtimes \mathbb{N} \rightarrow 0,
$$

the exact sequence at the level 1 (that we call so), and the quotient and closed ideal have the decompositions as follows:

$$
\begin{aligned}
0 \rightarrow\left(\left(\otimes^{n-2} \mathfrak{F}\right) \otimes \mathbb{K} \otimes C(\mathbb{T})\right) \rtimes \mathbb{N} & \rightarrow\left(\left(\otimes^{n-1} \mathfrak{F}\right) \otimes C(\mathbb{T})\right) \rtimes \mathbb{N} \\
& \rightarrow\left(\left(\otimes^{n-2} \mathfrak{F}\right) \otimes C\left(\mathbb{T}^{2}\right)\right) \rtimes \mathbb{N} \rightarrow 0, \quad \text { and } \\
0 \rightarrow\left(\left(\otimes^{n-2} \mathfrak{F}\right) \otimes\left(\otimes^{2} \mathbb{K}\right)\right) \rtimes \mathbb{N} & \rightarrow\left(\left(\otimes^{n-1} \mathfrak{F}\right) \otimes \mathbb{K}\right) \rtimes \mathbb{N} \\
& \rightarrow\left(\left(\otimes^{n-2} \mathfrak{F}\right) \otimes C(\mathbb{T}) \otimes \mathbb{K}\right) \rtimes \mathbb{N} \rightarrow 0,
\end{aligned}
$$

the exact sequences at the level 2. Inductively, the exact sequences at the level $k(1 \leq k \leq n)$ have quotients and closed ideals that are given by

$$
\left(\left(\otimes^{n-k} \mathfrak{F}\right) \otimes\left(\otimes^{l} \mathbb{K}\right) \otimes C\left(\mathbb{T}^{k-l}\right)\right) \rtimes \mathbb{N}
$$

$(0 \leq l \leq k)$ where $\otimes^{0} \mathbb{K}=\mathbb{C}$ and $C\left(\mathbb{T}^{0}\right)=\mathbb{C}$. In particular, the exact sequences at the level $n$ have quotients and closed ideals that are given by

$$
\left(\left(\mathbb{K}^{l}\right) \otimes C\left(\mathbb{T}^{n-l}\right)\right) \rtimes \mathbb{N} \cong \begin{cases}\mathbb{K} \otimes C(\mathbb{T}) & (l=n), \\ \mathbb{K} \otimes\left(C\left(\mathbb{T}^{n-l}\right) \rtimes \mathbb{Z}\right) & (1 \leq l \leq n-1), \\ C\left(\mathbb{T}^{n}\right) \rtimes \mathbb{Z} & (l=0),\end{cases}
$$

and $C\left(\mathbb{T}^{n-l}\right) \rtimes \mathbb{Z}$ is isomorphic to the group $C^{*}$-algebra of the (generalized) discrete ax $+b$ group $\mathbb{Z}^{n-l} \rtimes \mathbb{Z}$. 
Proof. Note that $\left(\left(\mathbb{K}^{l}\right) \otimes C\left(\mathbb{T}^{n-l}\right)\right) \rtimes \mathbb{N}$ is isomorphic to the following:

$$
\left(\left(\mathbb{K}^{l}\right) \otimes C\left(\mathbb{T}^{n-l}\right)\right) \rtimes \mathbb{Z} \cong\left(\mathbb{K}^{l}\right) \otimes\left(C\left(\mathbb{T}^{n-l}\right) \rtimes \mathbb{Z}\right) \cong \mathbb{K} \otimes\left(C\left(\mathbb{T}^{n-l}\right) \rtimes \mathbb{Z}\right) .
$$

Proposition 2.11. The K-theory groups of $C^{*}\left(H_{n, 1}\right)$ are obtained as:

$$
K_{j}\left(C^{*}\left(H_{n, 1}\right)\right) \cong \mathbb{Z} \quad(j=0,1) .
$$

Proof. Since $C^{*}\left(H_{n, 1}\right)=\left(\otimes^{n} \mathfrak{F}\right) \rtimes_{\alpha} \mathbb{N}$, we have the Pimsner-Voiculescu sequence:

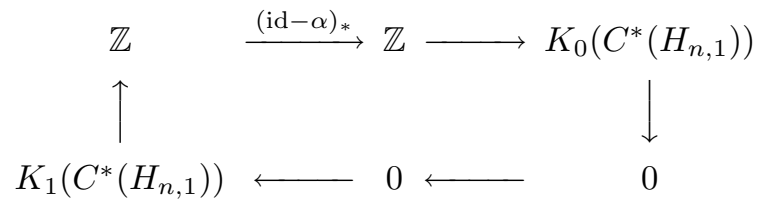

where $K_{0}\left(\otimes^{n} \mathfrak{F}\right) \cong \mathbb{Z}$ and $K_{1}\left(\otimes^{n} \mathfrak{F}\right) \cong 0$ by the Künneth formula (see [2]). Since the map (id $\left.-\alpha\right)_{*}$ is trivial, where id is the identity map on $\otimes^{n} \mathfrak{F}$, we obtain $K_{j}\left(C^{*}\left(H_{n, 1}\right)\right) \cong \mathbb{Z}$ for $j=0,1$.

Theorem 2.12. The stable rank of $C^{*}\left(H_{n, 1}\right)$ is $\lceil\lfloor(n+1) / 2\rfloor / 2\rceil+1$ if $n \neq 4 m$, and if $n=4 m$, then $m+1 \leq \operatorname{sr}\left(C^{*}\left(H_{n, 1}\right)\right) \leq m+2$.

The connected stable rank of $C^{*}\left(H_{n, 1}\right)$ is estimated as:

$$
2 \leq \operatorname{csr}\left(C^{*}\left(H_{n, 1}\right)\right) \leq\lceil\lfloor(n+2) / 2\rfloor / 2\rceil+1 .
$$

Proof. Using the structure obtained for $C^{*}\left(H_{n, 1}\right)$ above and [10, Theorems 4.3, 4.4, and 4.11] repeatedly as before for estimating the stable rank, we obtain that $\operatorname{sr}\left(C^{*}\left(H_{n, 1}\right)\right)$ is estimated by

$$
\left\{\begin{array}{l}
\operatorname{sr}(\mathbb{K} \otimes C(\mathbb{T}))=1, \\
\operatorname{sr}\left(\mathbb{K} \otimes\left(C\left(\mathbb{T}^{n-l}\right) \rtimes \mathbb{Z}\right)\right) \leq 2, \quad \operatorname{csr}\left(\mathbb{K} \otimes\left(C\left(\mathbb{T}^{n-l}\right) \rtimes \mathbb{Z}\right)\right) \leq 2, \\
\operatorname{sr}\left(C\left(\mathbb{T}^{n}\right) \rtimes \mathbb{Z}\right), \quad \text { and } \quad \operatorname{csr}\left(C\left(\mathbb{T}^{n}\right) \rtimes \mathbb{Z}\right)
\end{array}\right.
$$

$(1 \leq l \leq n-1)$. Note that $C\left(\mathbb{T}^{n}\right) \rtimes \mathbb{Z} \cong C^{*}\left(\mathbb{Z}^{n} \rtimes \mathbb{Z}\right)$. By the stable rank and connected stable rank formulae in [14, Remark 3.4] with a correction (see the remark above) we have

$$
\begin{aligned}
& \lceil\lfloor(n+1) / 2\rfloor / 2\rceil+1 \leq \operatorname{sr}\left(C^{*}\left(\mathbb{Z}^{n} \rtimes \mathbb{Z}\right)\right) \leq\lceil\lfloor(n+2) / 2\rfloor / 2\rceil+1, \\
& \text { and } \quad \operatorname{csr}\left(C^{*}\left(\mathbb{Z}^{n} \rtimes \mathbb{Z}\right)\right) \leq\lceil\lfloor(n+2) / 2\rfloor / 2\rceil+1
\end{aligned}
$$

where the stable rank estimate becomes equality if $n \neq 4 m$. Therefore, we obtain the stable rank estimates as in the statement.

On the other hand, using the structure obtained for $C^{*}\left(H_{n, 1}\right)$ above and [13, Theorem 3.9] repeatedly as before for estimating the connected stable rank, we obtain that $\operatorname{csr}\left(C^{*}\left(H_{n, 1}\right)\right)$ is estimated by

$$
\left\{\begin{array}{l}
\operatorname{csr}(\mathbb{K} \otimes C(\mathbb{T})) \leq 2 \\
\operatorname{csr}\left(\mathbb{K} \otimes\left(C\left(\mathbb{T}^{n-l}\right) \rtimes \mathbb{Z}\right)\right) \leq 2 \quad(1 \leq l \leq n-1), \\
\operatorname{csr}\left(C\left(\mathbb{T}^{n}\right) \rtimes \mathbb{Z}\right) .
\end{array}\right.
$$


Hence, it follows that $\operatorname{csr}\left(C^{*}\left(H_{n, 1}\right)\right) \leq\lceil\lfloor(n+2) / 2\rfloor / 2\rceil+1$. Since $K_{1}$-group of $C^{*}\left(H_{n, 1}\right)$ is not trivial as shown above, we have $\operatorname{csr}\left(C^{*}\left(H_{n, 1}\right)\right) \geq 2$ (cf. [4, Corollary 1.6]).

Remark. As a note, the stable rank and connected stable rank of $\otimes^{n} \mathfrak{F}$ are estimated as:

$$
\begin{aligned}
& \max \left\{2, \operatorname{sr}\left(C\left(\mathbb{T}^{n}\right)\right)\right\} \max \{2,\lfloor n / 2\rfloor+1\} \leq \\
& \operatorname{sr}\left(\otimes^{n} \mathfrak{F}\right) \leq \operatorname{csr}\left(C\left(\mathbb{T}^{n}\right)\right) \leq\lceil(n+1) / 2\rceil+1, \quad \text { and } \\
& \operatorname{csr}\left(\otimes^{n} \mathfrak{F}\right) \leq \operatorname{csr}\left(C\left(\mathbb{T}^{n}\right)\right) \leq\lceil(n+1) / 2\rceil+1
\end{aligned}
$$

using the structure of $\otimes^{n} \mathfrak{F}$ as above.

\section{Real rank}

Theorem 3.1. For an exact sequece of $C^{*}$-algebras: $0 \rightarrow \mathfrak{I} \rightarrow \mathfrak{A} \rightarrow \mathfrak{A} / \mathfrak{I} \rightarrow 0$, we obtain the following real rank estimate:

$$
\operatorname{RR}(\mathfrak{A}) \leq \max \{\operatorname{RR}(\mathfrak{I}), \operatorname{RR}(\mathfrak{A} / \mathfrak{I}), \operatorname{csr}(\mathfrak{A} / \mathfrak{I})-1\}
$$

Proof. Let $n$ be the maximum given above. We may assume that $n$ is finite since if it is infinite, the estimate is automatic. Let $\left(a_{j}\right)_{j=0}^{n}$ be an element of $\mathfrak{A}^{n+1}$ with $a_{j}=a_{j}^{*}$. Let $U$ be an open neighborhood of $\left(a_{j}\right)_{j=0}^{n}$. Let $\pi: \mathfrak{A} \rightarrow \mathfrak{A} / \mathfrak{I}$ be the quotient map. Write $\pi$ for the map $\mathfrak{A}^{n+1} \rightarrow(\mathfrak{A} / \mathfrak{I})^{n+1}$ extended by $\pi$. Then there exists an element $\left(b_{j}^{\prime}\right)_{j=0}^{n}$ of the intersection $\pi(U) \cap L_{n+1}(\mathfrak{A} / \mathfrak{I})_{s a}$ such that $\left(\pi\left(a_{j}\right)\right)_{j=0}^{n}$ is approximated closely by $\left(b_{j}^{\prime}\right)_{j=0}^{n}$. Note that $L_{n+1}(\mathfrak{A} / \mathfrak{I})_{s a}$ is a subest of $L_{n+1}(\mathfrak{A} / \mathfrak{I})$. Since $\operatorname{csr}(\mathfrak{A} / \mathfrak{I}) \leq n+1$, there exists an invertible matrix $S^{\prime}$ of $G L_{n+1}(\mathfrak{A} / \mathfrak{I})_{0}$ the connected component of $G L_{n+1}(\mathfrak{A} / \mathfrak{I})$ with the identity matrix such that $S^{\prime}\left(b_{j}^{\prime}\right)_{j=0}^{n}=(1,0, \cdots, 0)$. Then there exists a lift $S\left(b_{j}\right)_{j=0}^{n}$ of $S^{\prime}\left(b_{j}^{\prime}\right)_{j=0}^{n}$, where $S \in G L_{n+1}(\mathfrak{A})_{0}$ and $\left(b_{j}\right) \in U$ such that $S\left(b_{j}\right)_{j=0}^{n}=\left(1+c_{0}, c_{1}, \cdots, c_{n}\right) \in$ $\left(\mathfrak{I}^{\sim}\right)^{n+1}$ with each $c_{j} \in \mathfrak{I}$. Set $S\left(b_{j}\right)_{j=0}^{n}+\left(S\left(b_{j}\right)_{j=0}^{n}\right)^{*}=\left(d_{j}\right)_{j=0}^{n} \in\left(\mathfrak{I}^{\sim}\right)_{s a}^{n+1}$. Since $\operatorname{RR}(\mathfrak{I}) \leq n$, we may assume that $\left(d_{j}\right)_{j=0}^{n} \in L_{n+1}\left(\mathfrak{I}^{\sim}\right)_{s a}$, where $\mathfrak{I}^{\sim}$ is the unitization of $\mathfrak{I}$. Indeed, the set of the elements $\left(d_{j}\right)_{j=0}^{n} \in \mathfrak{A}_{s a}^{n+1}$ such that $S\left(b_{j}\right)_{j=0}^{n}$ is mapped by $\pi$ to an open neighborhood of $(1,0, \cdots 0)$ is open relative to $\left(\mathfrak{I}_{s a}^{\sim}\right)^{n+1}$, i.e., its intersection with $\left(\mathfrak{I}_{s a}^{\sim}\right)^{n+1}$ is open in $\left(\mathfrak{I}_{s a}^{\sim}\right)^{n+1}$ since any element of $\mathfrak{I}^{n+1}$ is mapped to $(0)_{j=0}^{n}$ by $\pi$. Note that $L_{n+1}\left(\mathfrak{I}^{\sim}\right)_{s a} \subset L_{n+1}\left(\mathfrak{A}^{\sim}\right)$, where $\mathfrak{A}^{\sim}=\mathfrak{A}$ if $\mathfrak{A}$ is unital and $\mathfrak{A}^{\sim}$ is the unitization of $\mathfrak{A}$ if $\mathfrak{A}$ is non-unital. Note also that $\left(b_{j}\right)_{j=0}^{n}+S^{-1}\left(S\left(b_{j}\right)_{j=0}^{n}\right)^{*}=S^{-1}\left(d_{j}\right)_{j=0}^{n} \in L_{n+1}\left(\mathfrak{A}^{\sim}\right)$ that is invariant under multiplication by elements of $G L_{n+1}(\mathfrak{A})_{0}$. By taking a deformation of $S$ (or $\left.S^{-1}\right)$ to the identity matrix in $G L_{n+1}(\mathfrak{A})_{0}$, it is concluded that $\left(b_{j}\right)_{j=0}^{n}+\left(b_{j}^{*}\right)_{j=0}^{n}$ is in $L_{n+1}(\mathfrak{A})_{s a}$, and belongs to $U$, as desired.

Remark. This real rank estimate for extensions of $C^{*}$-algebras, obtained above will be very useful for computing the real rank of the extensions, as shown below. The estimate corresponds to the following of Rieffel [10, Theorem 4.11]:

$$
\operatorname{sr}(\mathfrak{A}) \leq \max \{\operatorname{sr}(\mathfrak{I}), \operatorname{sr}(\mathfrak{A} / \mathfrak{I}), \operatorname{csr}(\mathfrak{A} / \mathfrak{I})\}
$$

which is often used in Section 1.

Theorem 3.2. The real rank of the Toeplitz algebra $\mathfrak{F}$ is 1 . 
Proof. Since $0 \rightarrow \mathbb{K} \rightarrow \mathfrak{F} \rightarrow C(\mathbb{T}) \rightarrow 0$, the estimate obtained in the theorem above implies

$$
\mathrm{RR}(\mathfrak{F}) \leq \max \{\operatorname{RR}(\mathbb{K}), \operatorname{RR}(C(\mathbb{T})), \operatorname{csr}(C(\mathbb{T}))\}=\max \{0,1,1\}=1 .
$$

On the other hand, by [5, Theorem 1.4],

$$
\operatorname{RR}(\mathfrak{F}) \geq \max \{\operatorname{RR}(\mathbb{K}), \operatorname{RR}(C(\mathbb{T}))\}=1 .
$$

Remark. The same result as above is obtained as a corollary of [5, Theorem 1.2], which says that for an extension of $C^{*}$-algebras: $0 \rightarrow \mathbb{K} \rightarrow \mathfrak{A} \rightarrow \mathfrak{A} / \mathfrak{I} \rightarrow 0$, we have $\operatorname{RR}(\mathfrak{A})=\operatorname{RR}(\mathfrak{A} / \mathfrak{I})$. Also, the result [7, Proposition 1.6] implies that for an extension of $C^{*}$-algebras: $0 \rightarrow \mathfrak{I} \rightarrow \mathfrak{A} \rightarrow \mathfrak{A} / \mathfrak{I} \rightarrow 0$, we have

$$
\operatorname{RR}(\mathfrak{A}) \leq \max \{\operatorname{RR}(M(\mathfrak{I})), \operatorname{RR}(\mathfrak{A} / \mathfrak{I})\},
$$

where $M(\mathfrak{I})$ is the multiplier algebra of $\mathfrak{I}$. It follows from this estimate that $\mathfrak{F}$ has real rank 1 since $M(\mathbb{K}) \cong \mathbb{B}$ the $C^{*}$-algebra of bounded operators has real rank 0 [3]. However, the above estimate of $[7]$ is not always useful since it involves the multiplier algebra, and it is hard to know its structure in general so that it is difficult to estimate its real rank in general.

Theorem 3.3. The real rank of $C^{*}(\mathbb{Z} \rtimes \mathbb{Z})$ of the discrete $a x+b$ group is 1 .

Proof. It is shown in [14] that $C^{*}(\mathbb{Z} \rtimes \mathbb{Z})$ has a composition series $\left\{\mathfrak{I}_{j}\right\}_{j=1}^{3}$ of closed ideals, with $\mathfrak{I}_{3}=C^{*}(\mathbb{Z} \rtimes \mathbb{Z})$ such that

$$
\begin{aligned}
& \mathfrak{I}_{3} / \mathfrak{I}_{2} \cong C(\mathbb{T}) \oplus C(\mathbb{T}), \quad \mathfrak{I}_{2} / \mathfrak{I}_{1} \cong C_{0}(\mathbb{R}) \otimes M_{2}(\mathbb{C}), \\
& \text { and } \quad \mathfrak{I}_{1} \cong C_{0}\left(\mathbb{R}^{2}\right) \otimes M_{2}(\mathbb{C})
\end{aligned}
$$

Using the real rank estimate obtained above,

$$
\begin{aligned}
& \operatorname{RR}\left(\mathfrak{I}_{3}\right) \leq \max \left\{\operatorname{RR}\left(\mathfrak{I}_{2}\right), \operatorname{RR}\left(\mathfrak{I}_{3} / \mathfrak{I}_{2}\right), \operatorname{csr}\left(\mathfrak{I}_{3} / \mathfrak{I}_{2}\right)-1\right\}, \quad \text { and } \\
& \operatorname{RR}\left(\mathfrak{I}_{2}\right) \leq \max \left\{\operatorname{RR}\left(\mathfrak{I}_{1}\right), \operatorname{RR}\left(\mathfrak{I}_{2} / \mathfrak{I}_{1}\right), \operatorname{csr}\left(\mathfrak{I}_{2} / \mathfrak{I}_{1}\right)-1\right\} .
\end{aligned}
$$

Also, by [5],

$$
\operatorname{RR}\left(\mathfrak{I}_{j}\right) \geq \max \left\{\operatorname{RR}\left(\mathfrak{I}_{j-1}\right), \operatorname{RR}\left(\mathfrak{I}_{j} / \mathfrak{I}_{j-1}\right)\right\}
$$

for $j=2,3$. By [13], $\operatorname{csr}\left(\mathfrak{I}_{3} / \mathfrak{I}_{2}\right)=\operatorname{csr}(C(\mathbb{T}))=2$. By [11, Theorem 4.7],

$$
\operatorname{csr}\left(C_{0}(\mathbb{R}) \otimes M_{2}(\mathbb{C})\right) \leq\left\lceil\left(\operatorname{csr}\left(C_{0}(\mathbb{R})\right)-1\right) / 2\right\rceil+1=2 .
$$

By [3, Proposition 1.1], $\operatorname{RR}\left(\mathfrak{I}_{3} / \mathfrak{I}_{2}\right)=\operatorname{RR}(C(\mathbb{T}))=1$. By [1],

$$
\begin{aligned}
\operatorname{RR}\left(C_{0}(\mathbb{R}) \otimes M_{2}(\mathbb{C})\right) & \geq\lceil\operatorname{dim}[0,1] /(2 \cdot 2-1)\rceil=1, \quad \text { while } \\
\operatorname{RR}\left(C_{0}(\mathbb{R}) \otimes M_{2}(\mathbb{C})\right) & \leq\left\lceil\operatorname{dim} S^{1} /(2 \cdot 2-1)\right\rceil=1, \quad \text { and } \\
\operatorname{RR}\left(C_{0}\left(\mathbb{R}^{2}\right) \otimes M_{2}(\mathbb{C})\right) & \geq\left\lceil\operatorname{dim}[0,1]^{2} /(2 \cdot 2-1)\right\rceil=1, \quad \text { while } \\
\operatorname{RR}\left(C_{0}\left(\mathbb{R}^{2}\right) \otimes M_{2}(\mathbb{C})\right) & \leq\left\lceil\operatorname{dim} S^{2} /(2 \cdot 2-1)\right\rceil=1,
\end{aligned}
$$

where $C([0,1]), C\left([0,1]^{2}\right)$ are quotients of $C_{0}(\mathbb{R}), C_{0}\left(\mathbb{R}^{2}\right)$ respectively, and $S^{1}, S^{2}$ are the one-point compactifications of $\mathbb{R}, \mathbb{R}^{2}$ (i.e., 1 and 2-dimensional spheres) respectively (see also [15] and [7, Proposition 5.1]). Therefore, it follows that $\operatorname{RR}\left(\mathfrak{I}_{j}\right)=1=\operatorname{RR}\left(\mathfrak{I}_{j} / \mathfrak{I}_{j-1}\right)$ for $j=1,2,3$. 
Theorem 3.4. The real rank of $C^{*}\left(H_{1,1}\right)$ is 1 .

Proof. Using the real rank estimate, the structure for $C^{*}\left(H_{1,1}\right)$ obtained above, and the theorem above, we obtain

$$
\begin{aligned}
& \operatorname{RR}\left(C^{*}(\mathbb{Z} \rtimes \mathbb{Z})\right)=1 \leq \operatorname{RR}\left(C^{*}\left(H_{1,1}\right)\right) \leq \\
& \max \left\{\operatorname{RR}(\mathbb{K} \otimes C(\mathbb{T})), \operatorname{RR}\left(C^{*}(\mathbb{Z} \rtimes \mathbb{Z})\right), \operatorname{csr}\left(C^{*}(\mathbb{Z} \rtimes \mathbb{Z})\right)-1\right\}=1,
\end{aligned}
$$

where $\operatorname{RR}(\mathbb{K} \otimes C(\mathbb{T})) \leq 1$ by [1].

Theorem 3.5. The real rank of $\otimes^{n} \mathfrak{F}$ is $n$.

Proof. By using the real rank estimate and the ( $n$-fold) structure for $\otimes^{n} \mathfrak{F}$, that can be obtained inductively as above from the structure for $\mathfrak{F}$, it follows that the real rank of $\otimes^{n} \mathfrak{F}$ is estimated by

$$
\left\{\begin{array}{l}
\operatorname{RR}\left(C\left(\mathbb{T}^{n}\right)\right)=n, \quad \operatorname{csr}\left(C\left(\mathbb{T}^{n}\right)\right)-1 \leq\lceil(n+1) / 2\rceil, \\
\operatorname{RR}\left(C\left(\mathbb{T}^{k}\right) \otimes \mathbb{K}\right) \leq 1, \quad \text { and } \quad \operatorname{csr}\left(C\left(\mathbb{T}^{k}\right) \otimes \mathbb{K}\right)-1 \leq 1
\end{array}\right.
$$

$(0 \leq k \leq n-1)$, where $C\left(\mathbb{T}^{0}\right)=\mathbb{C}$. The conclusion is deduced as before.

Theorem 3.6. The real rank of $C^{*}\left(\mathbb{Z}^{n} \rtimes \mathbb{Z}\right)$ of the generalized discrete $a x+b$ group is

$$
\operatorname{RR}\left(C^{*}\left(\mathbb{Z}^{n} \rtimes \mathbb{Z}\right)\right)=\lceil(n+1) / 3\rceil .
$$

Proof. It is shown in [14] that $C^{*}\left(\mathbb{Z}^{n} \rtimes \mathbb{Z}\right)$ has a composition series $\left\{\mathfrak{I}_{j}\right\}_{j=1}^{n+1}$ of closed ideals, with $\mathfrak{I}_{n+1}=C^{*}\left(\mathbb{Z}^{n} \rtimes \mathbb{Z}\right)$ such that $\mathfrak{I}_{n+1} / \mathfrak{I}_{n}$ is isomorphic to the $2^{n}$-fold direct sum of $C(\mathbb{T})$, and each subquotient $\mathfrak{I}_{j} / \mathfrak{I}_{j-1}$ for $1 \leq j \leq n$ (with $\mathfrak{I}_{0}=\{0\}$ ) is isomorphic to the combination ${ }_{n} C_{n-j-1}$ fold direct sum of the following extension $E_{j}$ :

$$
0 \rightarrow C_{0}\left(\mathbb{R}^{n-j+2}\right) \otimes\left(\oplus^{n-j+1} M_{2}(\mathbb{C})\right) \rightarrow E_{j} \rightarrow \oplus^{n-j+1} M_{2}(\mathbb{C}) \rightarrow 0 .
$$

Using the real rank estimate obtained above, we obtain

$$
\begin{aligned}
\operatorname{RR}\left(\mathfrak{I}_{n+1}\right) & \leq \max \left\{\operatorname{RR}\left(\mathfrak{I}_{n}\right), \operatorname{RR}(C(\mathbb{T})), \operatorname{csr}(C(\mathbb{T}))-1\right\}, \quad \text { and } \\
\operatorname{RR}\left(\mathfrak{I}_{j}\right) & \leq \max \left\{\operatorname{RR}\left(\mathfrak{I}_{j-1}\right), \operatorname{RR}\left(E_{j}\right), \operatorname{csr}\left(E_{j}\right)-1\right\}, \quad \text { and } \\
\operatorname{RR}\left(E_{j}\right) & \leq \max \left\{\operatorname{RR}\left(C_{0}\left(\mathbb{R}^{n-j+2}\right) \otimes M_{2}(\mathbb{C})\right), \operatorname{RR}\left(M_{2}(\mathbb{C})\right), \operatorname{csr}\left(M_{2}(\mathbb{C})\right)-1\right\} \\
& =\max \{\lceil(n-j+2) /(2 \cdot 2-1)\rceil, 0,0\}=\lceil(n-j+2) / 3\rceil .
\end{aligned}
$$

Also, by [5],

$$
\begin{aligned}
\operatorname{RR}\left(\mathfrak{I}_{n+1}\right) & \geq \max \left\{\operatorname{RR}\left(\mathfrak{I}_{n}\right), \operatorname{RR}(C(\mathbb{T}))\right\}, \quad \text { and } \\
\operatorname{RR}\left(\mathfrak{I}_{j}\right) & \geq \max \left\{\operatorname{RR}\left(\mathfrak{I}_{j-1}\right), \operatorname{RR}\left(E_{j}\right)\right\}, \quad \text { and } \\
\operatorname{RR}\left(E_{j}\right) & \geq\lceil(n-j+2) / 3\rceil .
\end{aligned}
$$

By [13],

$$
\operatorname{csr}\left(E_{j}\right) \leq \operatorname{csr}\left(C_{0}\left(\mathbb{R}^{n-j+2}\right) \otimes M_{2}(\mathbb{C})\right) \leq\lceil\lfloor(n-j+3) / 2\rfloor / 2\rceil+1
$$


Furthermore,

$$
\begin{aligned}
\operatorname{RR}\left(\Im_{j}\right) \geq \operatorname{RR}\left(\Im_{1}\right) & \geq \operatorname{RR}\left(C_{0}\left(\mathbb{R}^{n+1}\right) \otimes M_{2}(\mathbb{C})\right) \\
& \geq \operatorname{RR}\left(C\left([0,1]^{n+1}\right) \otimes M_{2}(\mathbb{C})\right) .
\end{aligned}
$$

By [1], it follows that

$$
\operatorname{RR}\left(C\left([0,1]^{n+1}\right) \otimes M_{2}(\mathbb{C})\right)=\lceil(n+1) /(2 \cdot 2-1)\rceil=\lceil(n+1) / 3\rceil .
$$

It follows that $\operatorname{RR}\left(\Im_{j}\right)=\lceil(n+1) / 3\rceil$ for $1 \leq j \leq n+1$.

Theorem 3.7. The real rank of $C^{*}\left(H_{n, 1}\right)$ is $\lceil(n+1) / 3\rceil$.

Proof. Using the structure obtained for $C^{*}\left(H_{n, 1}\right)$ above and the real rank esitame for extensions of $C^{*}$-algebras obtained above, we obtain that $\operatorname{RR}\left(C^{*}\left(H_{n, 1}\right)\right)$ is estimated by

$$
\left\{\begin{array}{l}
\operatorname{RR}(\mathbb{K} \otimes C(\mathbb{T})) \leq 1, \\
\operatorname{RR}\left(\mathbb{K} \otimes\left(C\left(\mathbb{T}^{n-l}\right) \rtimes \mathbb{Z}\right)\right) \leq 1, \quad \operatorname{csr}\left(\mathbb{K} \otimes\left(C\left(\mathbb{T}^{n-l}\right) \rtimes \mathbb{Z}\right)\right)-1 \leq 1, \\
\operatorname{RR}\left(C\left(\mathbb{T}^{n}\right) \rtimes \mathbb{Z}\right), \quad \text { and } \quad \operatorname{csr}\left(C\left(\mathbb{T}^{n}\right) \rtimes \mathbb{Z}\right)-1
\end{array}\right.
$$

$(1 \leq l \leq n-1)$. Note that $C\left(\mathbb{T}^{n}\right) \rtimes \mathbb{Z} \cong C^{*}\left(\mathbb{Z}^{n} \rtimes \mathbb{Z}\right)$. Moreover, we have obtained above that

$$
\begin{aligned}
& \operatorname{RR}\left(C^{*}\left(\mathbb{Z}^{n} \rtimes \mathbb{Z}\right)\right)=\lceil(n+1) / 3\rceil, \quad \text { and } \\
& \operatorname{csr}\left(C^{*}\left(\mathbb{Z}^{n} \rtimes \mathbb{Z}\right)\right) \leq\lceil\lfloor(n+2) / 2\rfloor / 2\rceil+1 .
\end{aligned}
$$

Therefore, we obtain the real rank formula as in the statement.

Remark. More applications by using the real rank estimate for extensions of $C^{*}$-algebras, obtained above, could be expected, when the extensions are given, where the real ranks of their closed ideal and quotients are computable. Furthermore, if a $C^{*}$-algebra has a composition series of closed ideals such that the real ranks of its subquotients are computable, then the real rank of the $C^{*}$-algebra can be estimated by using the real rank formula.

\section{A partial duality}

Definition 4.1. Let $\mathfrak{A} \rtimes_{\alpha} \mathbb{N}$ be the crossed product of a (unital) $C^{*}$-algebra $\mathfrak{A}$ by an action $\alpha$ of $\mathbb{N}$ by an isometry $s_{1}$. Define the second (or dual) crossed product of $\mathfrak{A} \rtimes_{\alpha} \mathbb{N}$ to be the crossed product $\mathfrak{A} \rtimes_{\alpha} \mathbb{N} \rtimes_{\beta} \mathbb{N}$ by a (dual) action $\beta$ of $\mathbb{N}$ such that $\beta$ is trivial on $\mathfrak{A}$, and $\beta$ on $C^{*}\left(s_{1}\right)$ generated by $s_{1}$ is implemented by an isometry $s_{2}$.

Proposition 4.2. The second crossed product $\mathfrak{B}=\mathfrak{A} \rtimes_{\alpha} \mathbb{N} \rtimes_{\beta} \mathbb{N}$ has the following decomposition:

$$
0 \rightarrow\left(\mathfrak{A} \rtimes_{\alpha} \mathbb{N}\right) \otimes \mathbb{K} \rightarrow \mathfrak{B} \rightarrow(\mathfrak{A} \otimes C(\mathbb{T})) \rtimes_{\alpha \otimes \beta} \mathbb{N} \rightarrow 0
$$

where the action $\beta$ on $C(\mathbb{T})$ is the adjoint action implemented by a unitary. 
Proof. Note that

$$
\mathfrak{A} \rtimes_{\alpha} \mathbb{N} \rtimes_{\beta} \mathbb{N} \cong\left(\mathfrak{A} \rtimes_{\text {id }} \mathbb{N}\right) \rtimes_{(\alpha, \beta)} \mathbb{N}
$$

where id is the identity action of the second $\mathbb{N}$, and the action $\beta$ on $C^{*}\left(s_{1}\right)$ is exchanged by the action $\alpha$ on $C^{*}\left(s_{2}\right)$ implemented by $s_{1}$. Since $\mathfrak{A} \rtimes_{\text {id }} \mathbb{N} \cong \mathfrak{A} \otimes \mathfrak{F}$, we have $(\alpha, \beta)=\alpha \otimes \beta$ a product action, so that there exists the following exact sequence:

$$
0 \rightarrow(\mathfrak{A} \otimes \mathbb{K}) \rtimes \mathbb{N} \rightarrow(\mathfrak{A} \otimes \mathfrak{F}) \rtimes \mathbb{N} \rightarrow(\mathfrak{A} \otimes C(\mathbb{T})) \rtimes \mathbb{N} \rightarrow 0
$$

where the action $\beta$ on $C(\mathbb{T})$ becomes a unitary action. Furthermore, we obtain

$$
(\mathfrak{A} \otimes \mathbb{K}) \rtimes \mathbb{N} \cong(\mathfrak{A} \rtimes \mathbb{N}) \otimes \mathbb{K}
$$

Remark. K-theory for the closed ideal and quotient in the above exact sequence for the second crossed product $\mathfrak{B}$ by $\mathbb{N}$ can be computed by the Pimsner-Voiculescu exact sequence:

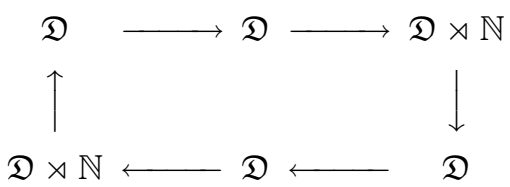

where $\mathfrak{D} \rtimes \mathbb{N}$ is the crossed product of a unital $C^{*}$-algebra $\mathfrak{D}$ by an action of $\mathbb{N}$ by a corner endomorphism ([12]). Furthermore, by using the six-term exact sequence for extensions of $C^{*}$-algebras, K-theory of $\mathfrak{B}$ can be determined when K-theory for $\mathfrak{A}$ is computable.

Example 4.3. Let $O_{n}$ be the Cuntz algebra generated by $n$ isometries $s_{j}$ such that $\sum_{j=1}^{n} s_{j} s_{j}^{*}=1$ (see [2] for instance). It is well known that $O_{n} \cong M_{n \infty} \rtimes_{\alpha} \mathbb{N}$ where $M_{n \infty}$ is the UHF algebra of type $n^{\infty}$, that is an inductive limit of tensor products $\otimes^{k} M_{n}(\mathbb{C})\left(\cong M_{n^{k}}(\mathbb{C})\right)$. Then the second crossed product $\mathfrak{B}=M_{n \infty} \rtimes \mathbb{N} \rtimes_{\beta} \mathbb{N} \cong O_{n} \rtimes \mathbb{N}$ has the decomposition:

$$
0 \rightarrow O_{n} \otimes \mathbb{K} \rightarrow \mathfrak{B} \rightarrow\left(M_{n \infty} \otimes C(\mathbb{T})\right) \rtimes_{\alpha \otimes \beta} \mathbb{N} \rightarrow 0 .
$$

Note that $\operatorname{sr}\left(O_{n} \otimes \mathbb{K}\right)=2$ since $\operatorname{sr}\left(O_{n}\right)=\infty$ by [10, Proposition 6.5]. Also, by [10, Theorem 5.1], $M_{n \infty} \otimes C(\mathbb{T})$ has stable rank 1 because it can be viewed as an inductive limit of matrix algebras over $C(\mathbb{T})$. However, $\mathfrak{B}$ has stable rank $\infty$ since the quotient of $\mathfrak{B}$ has stable rank $\infty$ because the quotient has $O_{n}$ as a quotient. Indeed, $M_{n \infty} \otimes \mathbb{C} 1$ is invariant under the action of $\mathbb{N}$. This shows that the second crossed product $\mathfrak{B}$ can not be stable.

Received: March 2009. Revised: July 2009.

\section{References}

[1] E.J. Beggs and D.E. Evans, The real rank of algebras of matrix valued functions, Internat. J. Math. 2 (1991), 131-138.

[2] B. Blackadar, K-theory for Operator Algebras, Second Edition, Cambridge, (1998). 
[3] L.G. Brown and G.K. Pedersen, $C^{*}$-algebras of real rank zero, J. Funct. Anal. 99 (1991), 131-149.

[4] N. Elhage Hassan, Rangs stables de certaines extensions, J. London Math. Soc. 52 (1995), 605-624.

[5] N. Elhage Hassan, Rang réel de certaines extensions, Proc. Amer. Math. Soc. 123 (1995), 3067-3073.

[6] G.J. Murphy, $C^{*}$-algebras and Operator theory, Academic Press, (1990).

[7] M. Nagisa, H. Osaka And N.C. Phillips, Ranks of algebras of continuous $C^{*}$-algebra valued functions, Canad. J. Math. 53 (2001), 979-1030.

[8] V. Nistor, Stable rank for a certain class of type I $C^{*}$-algebras, J. Operator Theory 17 (1987), 365-373.

[9] G.K. Pedersen, $C^{*}$-Algebras and their Automorphism Groups, Academic Press (1979).

[10] M.A. Rieffel, Dimension and stable rank in the $K$-theory of $C^{*}$-algebras, Proc. London Math. Soc. 46 (1983), 301-333.

[11] M.A. RIEfFEL, The homotopy groups of the unitary groups of non-commutative tori, J. Operator Theory 17 (1987), 237-254.

[12] M. Rørdam, Classification of certain infinite simple $C^{*}$-algebras, J. Funct. Anal. 131, (1995), 415-458.

[13] A.J-L. Sheu, A cancellation theorem for projective modules over the group $C^{*}$-algebras of certain nilpotent Lie groups, Canad. J. Math. 39 (1987), 365-427.

[14] T. Sudo, The structure of group $C^{*}$-algebras of some discrete solvable semi-direct products, Hokkaido Math. J. XXXIII(33), no. 3, (2004), 587-606.

[15] T. Sudo, Real rank estimate by hereditary $C^{*}$-subalgebras by projections, Math. Scand. 100 (2007), 361-367. 